\title{
Design and implementation of the monitoring system of fowlery temperature and humidity environment based on ZigBee
}

\author{
Peng Zhanwu, ${ }^{1, a}$, Wang Xue ${ }^{2, b}$
}

\author{
${ }^{1} 1$ Information technology teaching and management center, Jilin Agricultural University, \\ Changchun 130118, China \\ ${ }^{2}$ Institute of Scientific and Technical Information of Jilin, Changchun 130021,China \\ a13908738@qq.com, b21211430@qq.com
}

Keywords: ZigBee, Wireless Sensor Network (WSN), Environmental Monitoring

\begin{abstract}
A ZigBee wireless sensor network was designed and implemented, to monitor the temperature and humidity changes of the fowlery. Breaking the various malpractices of the traditional arrangement of wire monitoring points. After the actual test, results showed that using the ZigBee wireless sensor network system to monitor the environment temperature and humidity of the fowlery had the advantages of low cost, low power consumption, layout of flexible, accurate measurement and stable operation etc., improved the automation level of scientific culture.
\end{abstract}

\section{Introduction}

In China, poultry meat, eggs are always important dishes on the table, with the improvement of people's living standard, demand of poultry products is also increasing, people not only to eat, but also to the increasingly strong demand to the green pollution-free poultry products. In recent years, due to the emergence of avian influenza and other diseases is make people talk about "birds" pale, which of the livestock and poultry industry proposed higher requirements, on the one hand to increase the yield of livestock and poultry products, on the other hand the product quality requirements more stringent. Forcing some traditional livestock and poultry breeding enterprises to change the status quo, and actively seek modern technology, to effectively monitor the environment of breeding, in order to improve the yield and quality of livestock and poultry products.

The study showed that the growth environment directly affects the health of livestock and poultry, especially in the closed fowlery. If you can't effectively control the breeding environment, the growth, development and reproduction of livestock and poultry will lead to slow growth, low egg production, and even spread diseases etc.[1]

The research took the closed fowlery as the research object, used of computer technology, network communication technology and sensor technology, designed a kind of temperature and humidity data acquisition system based on ZigBee. The system was a distributed monitoring system composed by a number of nodes. The nodes were free distribution, real-time sensing temperature and humidity changes, wireless communication and self-networking capability. This system monitored 
and controlled the temperature and humidity changes of the chicken house microclimate environment, to supply favorable conditions for the chicken to growth and development, effective control of the occurrence and spread of diseases, improve the yield and quality of livestock and poultry products.

\section{System composition principle and general design}

ZigBee working principle. ZigBee is a low speed and short distance transmission wireless network protocol, with low power, low cost, high reliability and other characteristics, in recent years it has been widely used in various fields of data acquisition, environmental monitoring and other applications. MAC layer and PHY layer of ZigBee protocol using IEEE802.15.4 protocol standards, and its main working band $2.4 \mathrm{G}$ for the global free, free registration frequency band, the transmission rate is $250 \mathrm{kbps}[2]$.

The biggest characteristic of ZigBee network is that it has a certain self-organization and self-healing ability ${ }^{[2]}$. In the case of no human intervention, the network node can perceive the existence of other nodes, and determine the connection relationship, which constitute a structured network. Also in the circumstances of add or delete a node, node failures or position change, the network can achieve self-repair and topological structure of network is adjusted to ensure that the whole system will still be able to normal operation.

General design. General size of the henhouse length at about 100 meters, about 5000-8000 chickens and considering of both spatial span and measurement node signal strength, arranging of 20 nodes are appropriate, from the henhouse within 50 meters configurations of a base station and a general computer, the station is a sink node, is responsible for receiving the data sent from the sensor nodes, computer is mainly used for data storage and processing. The general design is shown in Fig1.

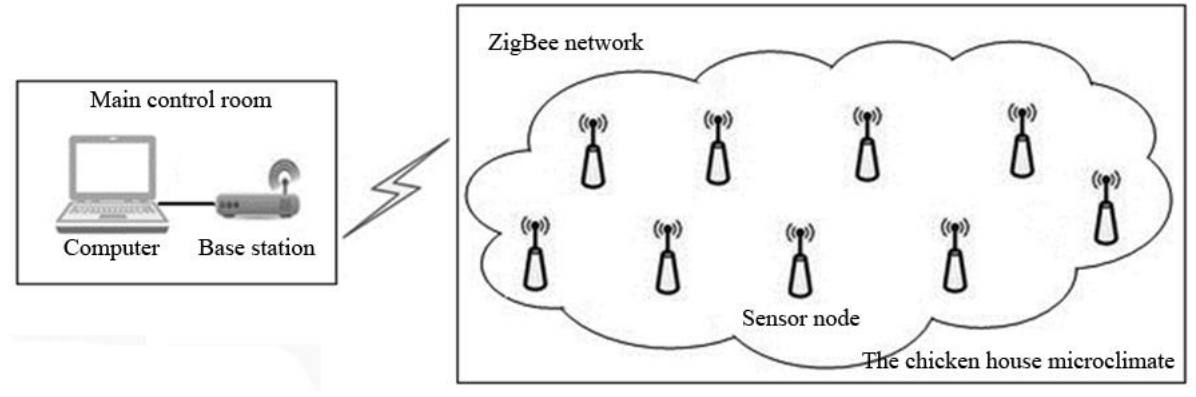

Fig. 1 system general design

The whole system consists of the chicken house microclimate and main control room, which henhouse environment as the monitoring object, arranged a number of ZigBee wireless nodes, each node respectively integrated temperature and humidity sensor, which is responsible for collection of environmental parameters, node self-organizing network according to the ZigBee protocol, mutual communication, transmitting data to the sink node, that is, the base station. A base station and a host machine constitute the main control room, the data received from base station would be aggregated, are connected through a serial port and PC, data would be conversed to binary, real-time display parameter change of the henhouse environment, and storage historical data to computer hard drives, and upload to the server, for query. 


\section{Hardware design}

ZigBee node. ZigBee node is the core design of the hardware components, function in accordance with the ZigBee network protocol is to self-organize network, the communication between the nodes, the transmission of data. Taking the module DSU1115-0601 as network nodes and its main components CC2530 is produced by American TI ZigBee wireless chip, with typical ZigBee wireless transmission characteristics, integrated the 8051 single chip and a RF transceiver, the working voltage is $3.6 \mathrm{~V}$ and has $256 \mathrm{~Kb}$ flash memory. CC2530 also has a strong anti-interference ability and scalability, the data can be achieved A/D conversion and information transfers between the nodes, and be able to communicate with the host computer[3]. The organizational structure of CC2530 is shown in Fig 2.

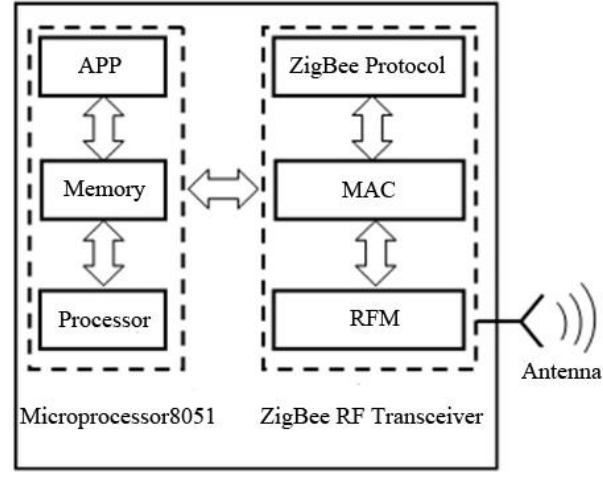

Fig 2. composition structure of CC2530

Temperature and humidity sensor. The AM2305 digital temperature and humidity sensor is a kind of composite sensor, which is used to calibrate the digital signal output. It uses a special digital acquisition module and temperature and humidity sensing technology, to ensure the system requirement of high reliability and high stability, the sensor includes a capacitive moisture element and DS18B20 emperature measuring element and was connected with a high performance 8-bit single chip. The working voltage of AM 2305 is 3.3-6V, which is consistent with the working voltage of the ZigBee module, with two AA batteries can work for a long time, and the measurement accuracy for temperature was $\pm 0.2^{\circ} \mathrm{C}$, for humidity was $\pm 2 \% \mathrm{RH}$, enough to meet the requirements of the fowlery environment.

\section{Software design}

The software design of the system is mainly to realize the function of hardware and provide a visual data display window, which can be divided into the underlying software and the upper computer software.

The underlying software design is mainly on writing the ZigBee-Stack-CC2530 protocol stack, and CC2530 initialization, form ZigBee network and join the network, send commands and receiving instructions, return data to the sink node and other operation [4].

PC software design is using programming language to develop a visualization application platform. Binary conversion of the data received by the computer serial port, and stored in the 
database, software interface can real-time display the changes of measurement data, can also be achieved historical data export from the database.

PC software was developed by VB, measurement data stored in the ACCESS database, the PC software development interface as shown in figure 3.

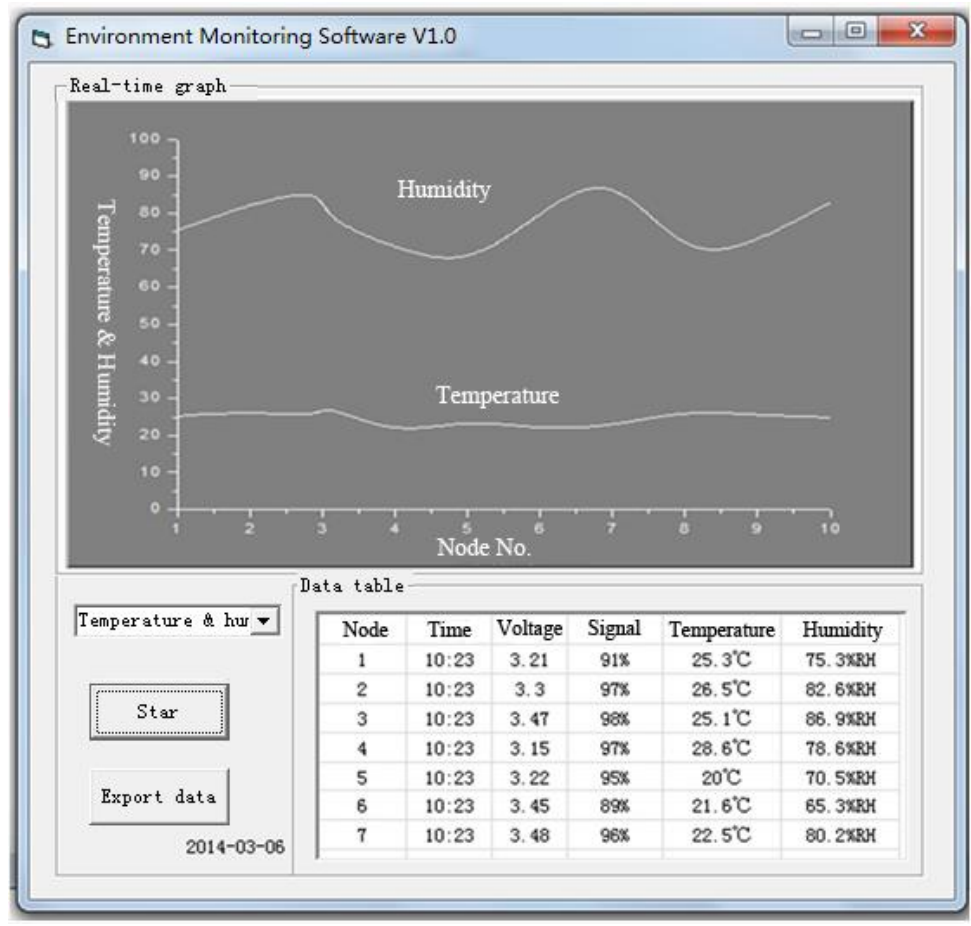

Fig. 3 PC software interface diagram

\section{System test}

System test in the 3rd Mingrui farm house of Changchun City, the henhouse length was about 50 meters, considering of both spatial span and measurement node signal strength, 10 ZigBee nodes were set, each node was integrated temperature and humidity sensor. After the power is added, the node starts to work, after initialization, the network is successful. The software in main control room, which 30 meters away from the fowlery was running normal, the data was collected after 1 minute, 10 nodes environment parameters and voltage value were measured, and also the signal strength information. Real time curves showed the changes of the temperature and humidity of the measuring points in the fowlery.

Each node received the data once every 1 minutes, after 2 hours of continuous operation, the data is more than 1200. According to the data, the system was accurate, stable, and the node voltage had no change, indicating that the ZigBee module was less energy consumption.

\section{Conclusions and Outlook}

The research took CC2530 chip as the core module, building the ZigBee wireless network, using AM2305 temperature and humidity sensors to monitor the fowlery environment, developing the PC 
software for receiving data, and real-time displayed the change curve of fowlery environment. The ZigBee wireless sensor network realized the monitoring of the fowlery environment, effectively solved the drawbacks past for the high cost wiring, inflexible distribution and maintenance difficulties. Improved the degree of automation of scientific farming, improved the environment of the breeding shed, reduced the disease spread among livestock and poultry caused by poor environment, and improved the yield and quality of products.

In addition to the big effect of temperature and humidity factors on the growth of livestock and poultry, a large number of toxic and harmful gas is another important factor that restrict the growth and development and cause infection of disease[5]. The next step also needs to connect both gas sensor and ZigBee module, monitoring of toxic and harmful gases in the breeding shed, to achieve a more comprehensive monitoring of environmental parameters data.

\section{Reference}

[1] Wang Sizhen, Jia Weixing. Henhouse temperature and humidity growth and production performance of broilers agricultural engineering technology [J].2002 (7): 20-21.

[2] Peng Yan. Research on Wireless Sensor Networks Based on [J]. ZigBee modern electronic technology.2011,34 (5): 49-51.

[3] Li Zhifang, Zhong Sheng Hong in IEEE 802.15.4 CC2530 wireless data transceiver design [J]. Microcontroller and embedded system application settings (7): 43-45.

[4] Wei Xing Zhu, Dai Yun, Huang Peng. Based on IOT nursing pigsty environment monitoring system of agricultural engineering [J]. Journal of, 2012,28 (11).243-247.

[5] Guo Xia, Shen Dili, Meng Yajun, etc.. Henhouse environment wireless monitoring system design and implementation [J]. China Agricultural Chemistry, 2013, 34 (5). 